\title{
Eery Space: Facilitating Virtual Meetings through Remote Proxemics
}

\author{
Maurício Sousa, Daniel Mendes, Alfredo Ferreira, João Madeiras Pereira, and \\ Joaquim Jorge \\ INESC-ID Lisboa / IST / Technical University of Lisbon \\ \{antonio.sousa, danielmendes, alfredo.ferreira\}@ist.utl.pt, \\ \{jap, jaj\}@inesc-id.pt
}

\begin{abstract}
Virtual meetings have become increasingly common with modern video-conference and collaborative software. While they allow obvious savings in time and resources, current technologies add unproductive layers of protocol to the flow of communication between participants, rendering the interactions far from seamless. In this work we introduce Remote Proxemics, an extension of proxemics aimed at bringing the syntax of co-located proximal interactions to virtual meetings. We propose Eery Space, a shared virtual locus that results from merging multiple remote areas, where meeting participants' are located side-by-side as if they shared the same physical location. Eery Space promotes collaborative content creation and seamless mediation of communication channels based on virtual proximity. Results from user evaluation suggest that our approach is sufficient to initiate proximal exchanges regardless of their geolocation, while promoting smooth interactions between local and remote people alike.
\end{abstract}

Keywords. Remote Proxemics, Virtual Meetings, Collaboration

\section{Introduction}

When people get together to discuss, they communicate in several manners, besides verbally. Hall [5] observed that space and distance between people (proxemics) impact interpersonal communication. While this has been explored to leverage collaborative digital content creation [7], nowadays it is increasingly common for work teams to be geographically separated around the globe. In fact, through appropriate technology, it is possible to see and hear others, making it easier to communicate at a distance.

The newest videoconferencing and telepresence solutions support both common desktop environments and the latest mobile handheld technologies. However, despite considerable technological advances, remote users often feel neglected due to their limited presence [8]. Moreover, although verbal and visual communication occur naturally in virtual meetings, other modes of engagement, namely proximal interactions, have yet to be explored. This is unfortunate, since proxemics can enable many natural interactions obviating the need for cumbersome technology-induced protocol. 
In this work, we introduce Eery Space as a virtual construct to bring remote people together and mediate natural proxemics interactions between participants as if they were in the same physical place, a mechanism which we call Remote Proxemics. To this end, Eery Space allows us to merge different rooms into one virtual shared locus were people can meet, share resources and engage in collaborative tasks.

Building on the notion that people do not need hyper-realistic awareness devices, such as virtual avatars, to infer the presence of others [9] and engage in natural social behavior, Eery Space employs an iconic representation for remote people. Also, to facilitate virtual meetings, we propose novel techniques for person-to-person and person-to-device interactions. We adopt a multiple interactive surfaces environment, which comprises an ecosystem of handheld devices, wall-sized displays and projected floors.

\section{Eery Space}

We propose an approach to bring geographically distant people together into a common space, and to provide feedback for participants in a virtual meeting in order to be able to proximally interact. We call this common space Eery Space. Given that people are distributed across similar rooms in different locations, Eery Space attempts to consolidate these in a common virtual locus, while providing new opportunities for interaction and communication between participants. In this way, people equipped with personal handheld devices can meet and share resources regardless of where they are.

Instead of placing users in front of each other, as is typical of commercial applications and other research works [2, 3], we place both remote and local people side-byside, similar to Cohen et al. [4]. Unlike the common interactions with remote people using the mirror metaphor, Eery Space provides remote participants with a sense of being around local ones in a shared space. This creates and reinforces the model of a shared meeting area where proxemic interactions can take place. Moreover, each person gets assigned a definite position and a personal location within Eery Space. Allowing both local and remote people to collaborate by relating to their personal spaces strengthens the notion that everyone is treated similarly as if they were all physically co-located.

\subsection{Social Bubbles}

Hall's [5] model of proxemic distances dictates that when people are close to each other they can interact in specific ways. Within a proxemic social space, people do interact in a formal way, typical of a professional relationship. In contrast, the personal space is reserved for family and friends, and people can communicate quietly and comfortably. Yet, as described by Hall [5], these distances are dynamic. Friendship, social custom and professional acquaintanceship can decrease interpersonal distances [10]. We adapted these concepts to Eery Space, using a device we call Social Bubbles. 
Inside Eery Space, interactions are initiated by analyzing the distribution of people within the shared virtual space. People having a private conversation or involved in the same task usually get closer, and, therefore, we create social bubbles using a distance metaphor. People naturally create a bubble, where they can meet, share resources and engage in private conversation, by coming sufficiently close to each other. A social bubble appears through the intersection of two or more personal spaces, as depicted in Figure 1, implicitly capturing the intention of people to perform a collaborative task.

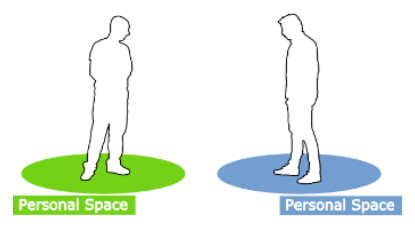

A

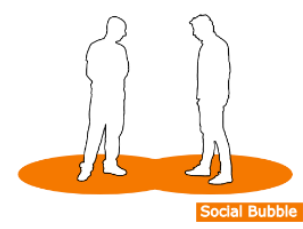

B

Fig. 1. Social Bubbles: (A) While distant from one another, (B) A social bubble happens when people's personal spaces intersect.

This formulation allows people motivated to initiate collaboration to easily create proximal interactions adopting a distance inside their social space, without needing to enter the other person's personal space. In our work, we considered personal space as a circle 0.6 meter in radius. Thus two people can create a social bubble by approaching the other within 1.2 meters. Destroying bubbles is analogous to creating them - a social bubbles ceases to exist when its participants move apart.

\subsection{Remote Proxemics}

Remote Proxemics aims to harness natural interactions that occur between co-located people and make these available to meeting participants who are not physically in the same room. In this way, all interactions within Eery Space work similarly for local and remote people. The success of our approach is to ensure that both local and remote people are always present and positioned side-by-side, so that participants can create social bubbles in a similar way, regardless of whether they are or not in the same room. Since Eery Space defines an environment with multiple people and devices, we have grouped these interactions into: person-to-person, involving people and their own mobile devices; and person-to-device, between people and shared devices.

Person-to-person Interactions: When people come together and create a social bubble, different tools become available to support collaborative tasks, as person-toperson interactions. These interactions include both the participants and their personal handheld devices, as depicted in Figure 2A. Since verbal communication is a key element to the success of virtual meetings, participants can both talk to and listen to other people inside their bubble. When people establish a social bubble, their handheld devices automatically open a communication channel to local and remote 
participants alike. This channel is closed when the bubble is destroyed. Similarly and simultaneously, if there is a shared visualization device, such as a wall display, the handheld devices of participants in the same social bubble can be synchronized to the common visualization. At this stage, participants can engage in a collaborative session around the shared visualization, either by discussing or by collaboratively creating content.

Person-to-device Interactions: The Eery Space may feature shared devices, such as wall displays and tabletops to support shared visualization and collaborative settings.



A

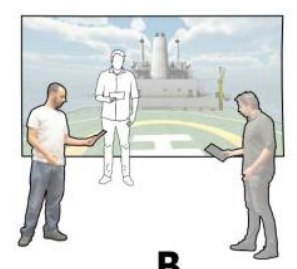

B

Fig. 2. Remote Proxemics: (A) Two local people and one remote (in white) engage in collaborative work in a social bubble. (B) The remote participant acts as the moderator.

In our work, we explored the latter kind, as shown in Figure 2 (B). Due to their large dimensions, these displays can provide a visualization surface to serve many people at the same time, and make the information under analysis accessible to all. Naturally, large displays should be located at the same virtual position across all remote areas that make up Eery Space, to ensure a consistent visualization to all participants. When a participant establishes a close proximity relationship with the display, he/she becomes moderator. In Eery Space, moderators have a special authority that allows them to control the common visualization on all shared displays, either local or remote, by mirroring actions performed on the handheld device. We define moderator space as the area within a distance of 1.5 meters away from the wall display, analogously to the place normally occupied by a person giving a talk to an audience. The role of moderator can only be handed over when the person assuming this role abandons the moderator space, leaving it available for another participant who wishes to take over. Furthermore, when a meeting participant becomes a moderator, a channel for speech communication is opened so that they can address all.

\section{Prototype}

We built a prototype system to prove that remote proxemics are possible and that Eery Space is an effective approach to manage interactions between participants as if all were in the same room. Our prototype employs multiple Microsoft Kinect depth cameras to locate people indoors in a non-intrusively fashion, while dealing with body occlusions. In this section, we describe the awareness techniques we implemented to 
provide appropriate feedback for interactions between participants. We opted to develop a scenario to design and review 3D CAD models in the oil and gas industry.

Floor Circles: In the Eery Space prototype, every local and remote participant has a representative projected circle on the room's floor, as depicted in Figure 3A. All circles are unique, corresponding to a single person, and are distinguished from each other by a name (the participant's identity) and the user's unique color. These circles track a person's position within Eery Space, in order to visually define the participant's personal space, thus making all people aware of others. Thus floor circles provide the necessary spatial information for participants to initiate and be aware of proximity interactions. In addition, projected circles depict a user's proxemic zones. The inner circle, with a

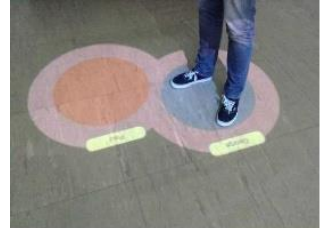

A

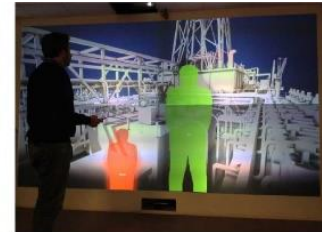

B

Fig. 3. Awareness techniques: (A) Floor circles. Remote and local users establish a social bubble. (B) Wall shadows depict two users. The larger shadow is the moderator.

radius of 0.3 meters, matches the participant's intimate space. The outer ring, depicts the personal space, with a radius of 0.6 meters. When people come together to start a social bubble, the circles on the floor depict the status of their Social Bubble, by matching the personal space color of the bubble participants, while maintaining the user's color in their intimate space. The social bubble receives a color which averages the color of its members. This guarantees that the bubble color is unique and unmistakably different from other content on the floor.

Intimate Space: We designed Eery Space keeping each person's personal locus in mind. Every user has their own space assured, even if they are not in the same physical room. To prevent users from invading another user's intimate space, we provide haptic feedback by vibrating their handheld device, when this happens. Participants can then quietly adjust their positions without interrupting the main meeting, since this technique does not use audio or visual cues. This way, each user's intimate space is preserved and made visible at all time to all participants, so that they can interact with it.

Wall Shadows: Additionally, and since we included large wall displays in our prototype, every person gets assigned a representative shadow on the wall display, distinguished by a name and a unique color, as shown in Figure 3B, similarly to the work of 
Apperley et al. [1]. This allows for a quick recognition of all meeting's participants. The location of the shadow reflects a distance from the person to the wall to give a sense of the spatial relationship between a person and the interactive surface. Wall shadows take in consideration an imaginary directional light source placed at infinity and oriented towards the wall display, with an inclination of 45 degrees. Thus, the nearest user to the wall will have a shadow covering more area than the others. A much larger shadow also makes clear who the moderator is.

\section{Evaluation}

We conducted a user evaluation to assess the interactions within the Eery Space, both with local and remote people. In our experiment, subjects were invited into the room with the main setup, while a remote user was in a room equipped with a lighter version with one Microsoft Kinect, one display showing the floor projection and a smartphone.

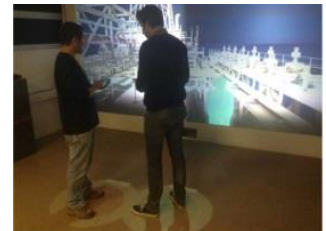

A

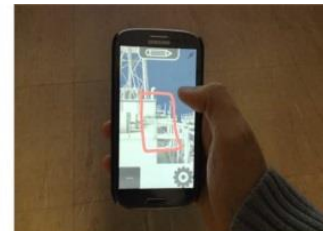

B

Fig. 4. Evaluation: (A) Test user interacting with the local participant during the evaluation session. (B) Handheld Client. User engaged in a sketch collaborative task.

Each participant received a smartphone running the handheld device client. Figure 4A shows our experimental setup. Participants were asked to perform collaborative sketching, (see Figure 4B) task with both local and remote people. To verify if subjects react to the presence of other remote people, their intimate space was purposely invaded to assess their reaction. The users were also asked to synchronize the visualization on the wall with the smartphone, by assuming the role of moderator.

All participants in this evaluation were randomly selected and were mainly students of our educational institution. The set of test users was comprised of 12 participants, one of which was female, and all with a college degree and with ages between 18 and 24 years old. Furthermore, every test user had no previous experience with our work.

\subsection{Results and Discussion}

Since the main objective of this evaluation was to demonstrate the feasibility of remote proxemics by maintaining an adequate level of awareness of the people that are remote, the analysis of the results is divided into Proxemics Analysis and Awareness Analysis. The data gathered were obtained from a Likert scale with 6 values. Table 1 summarizes the responses obtained from the questionnaire regarding those aspects. 
Proxemics Analysis: Participants' preferences regarding proxemics interactions are related to how easy it was to perform proximal interactions with both local and remote people, and also the ability to interact with the wall display. The latter, poses a conscious decision to become the moderator of the virtual meeting. The presented data suggests that it was easy to assume the role of moderator. According to the Wilcoxon Signed Ranks test, applied to the first and second questions $(\mathrm{Z}=-1.890, \mathrm{p}=0.059)$, there are no statistically significant differences between starting an interaction with the other participants, despite their local or remote statuses. This leads us to conclude that, in Eery Space, interacting with remote people is not that different than local interactions. This result is encouraging as it shows that remote proxemics are in fact possible and do not add obstacles in the course of virtual meetings. In the evaluation sessions, participants did not demonstrate any difficulty in repositioning themselves to establish social bubbles in the collaborative tasks, although three users took a little while (around five seconds) to remember how to become the moderator.

Awareness Analysis: For awareness, the data shows that people in the virtual meeting can relate to the presence of remote participants. Despite some dispersion in the data (question 5) user preferences suggestion that the location of remotely located people is

\begin{tabular}{|l|c|}
\hline It was easy to... & Median (IQR) \\
\hline ...control what is shown on the wall display. & $6(1.25)$ \\
\hline ...start an interaction with a local participant. & $6(0)$ \\
\hline ...start an interaction with a remote participant. & $6(1)$ \\
\hline ...see who is present at the meeting. & $6(0)$ \\
\hline ...see where each participant is. & $6(1.25)$ \\
\hline ...see who is controlling the wall display. & $6(0.25)$ \\
\hline ...see which participant I'm interacting with. & $6(0.25)$ \\
\hline ...see that I'm in the intimate space of another local participant. & $6(1)$ \\
\hline ...see that I'm in the intimate space of another remote participant. & $6(0)$ \\
\hline
\end{tabular}

Table 1. Questionnaire's results (median and interquartile range): proxemics overview (questions 1 to 3 ) and awareness overview (questions 4 to 10).

easily perceived. We can safely deduce that participants in the virtual meeting are always aware of the people involved. One of the requirements of our approach is the preservation of the intimate space of remote people. This design principle is required to impose their presence, while fostering remote interactions by establishing social bubbles. The Wilcoxon Signed Ranks test applied to the questions 6 and $7(\mathrm{Z}=0.000$, $\mathrm{p}=1.000)$ shows no statistically significant difference between local and remote people, suggesting that test users were aware when their intimate space intercepted others'. Curiously, while performing the collaborative task, three test users made a point of informing the remote participant of his infringement on their personal space during the smartphone-enabled conversation, before readjusting their position. Every subject changed their positions, during the intimate space invasion task, responding to the haptic feedback from the handheld device. Despite that, four users first complained that the remote participant was invading their intimate space, and only then proceeded 
to readjust their positions. In general, participants were aware of the presence of the remote participant and reacted accordingly.

\section{$5 \quad$ Conclusions and Future Work}

Results from our evaluation show the promise of Remote Proxemics, since we were able to achieve interactions between local and remote people. We believe that the work here described extends proxemic interactions to augment the presence of remote users in virtual collaborative settings to address commonly-raised concerns. Furthermore, our results apply even in the absence of commonly explored devices such as avatars and eye contact. We will assess whether f-formations [7] and gradual engagement [6] can enrich remote interactions in Eery Space.

\section{Acknowledgements}

This work was partially supported by the Portuguese Foundation for Science and Technology (FCT) through the projects TECTON-3D (PTDC/EEI-SII/3154/2012), CEDAR (PTDC/EIA-EIA/116070/2009), doctoral grant SFRH/BD/91372/2012 and by national funds through FCT with reference UID/CEC/50021/2013.

\section{References}

1. Apperley, M., McLeod, L., Masoodian, M., Paine, L., Phillips, M., Rogers, B., \& Thomson, K. (2003, February). Use of video shadow for small group interaction awareness on a large interactive display surface. In Proceedings of the Fourth Australasian user interface conference on User interfaces 2003-Volume 18 (pp. 81-90). Australian Computer Society, Inc.

2. Beck, S., Kunert, A., Kulik, A., \& Froehlich, B. (2013). Immersive group-to-group telepresence. Visualization and Computer Graphics, IEEE Transactions on, 19(4), 616625 .

3. Benko, H., Jota, R., \& Wilson, A. (2012, May). MirageTable: freehand interaction on a projected augmented reality tabletop. In Proceedings of the SIGCHI conference on human factors in computing systems (pp. 199-208). ACM.

4. Cohen, M., Dillman, K. R., MacLeod, H., Hunter, S., \& Tang, A. (2014, April). Onespace: Shared visual scenes for active freeplay. In Proceedings of the 32nd annual ACM conference on Human factors in computing systems (pp. 2177-2180). ACM.

5. E. T. Hall. The Hidden Dimension. Doubleday, (1966)

6. Marquardt, N., Ballendat, T., Boring, S., Greenberg, S., \& Hinckley, K. (2012, November). Gradual engagement: facilitating information exchange between digital devices as a function of proximity. In Proceedings of the 2012 ACM international conference on Interactive tabletops and surfaces (pp. 31-40). ACM.

7. Marquardt, N., Hinckley, K., \& Greenberg, S. (2012, October). Cross-device interaction via micro-mobility and f-formations. In Proceedings of the 25th annual ACM symposium on User interface software and technology (pp. 13-22). ACM.

8. L. Neyfakh. My day as a robot, May 2014, http://www.bostonglobe.com/ ideas/2014/05/10/day-robot/6UAMgmUFn0mZhoMS8vy0GK/story.html

9. Reeves, B and Nass, C.The Media Equation: How People Treat Computers, Tele-vision, and New Media Like Real People and Places. Cambridge Univ. Press, 1996.

10. Sommer, R. Personal space in a digital age. Handbook of environmental psychology, 647660 (2002) 